\title{
Hablar de niñez \\ no es hablar de pequeñeces Algunos desafíos para la política pública del Nivel Inicial en la Región de América Latina
}

\author{
To talk about childhood is no small thing. \\ A few challenges to early education public \\ policy in Latin America
}

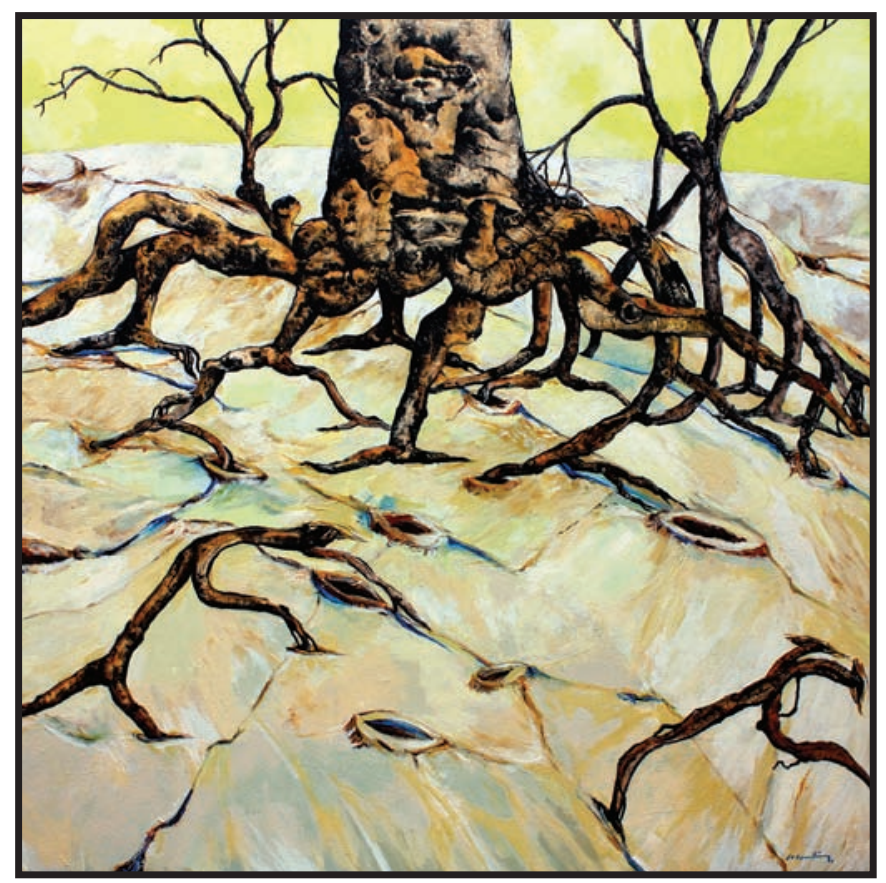

María Verónica Di Caudo*

Universidad Politécnica Salesiana mdicaudo@ups.edu.ec

Recibido: 3 de octubre de 2012 Aceptado: 19 de noviembre de 2012

Hablar del niño como sujeto significa pensar en una subjetividad en vías de constitución, a partir del discurso de los adultos, y que requiere que alguien le acerque al niño la lengua y la cultura, y que ofrezca espacios de protección que le posibiliten aprehenderla. Factores sociales, culturales, familiares y personales darán color propio a esta constitución.

(Mendioroz et al., 2009)

Sediento. $146 \times 146 \mathrm{~cm}$

* Directora y docente de la Carrera de Pedagogía, Sede Quito. Docente de Nivel Inicial. Licenciada en Ciencias de la Educación por la Universidad de Buenos Aires. Especialista en Planeamiento y Gestión de Educación a Distancia por la Universidad Católica de Brasilia. Master en Ciencias Sociales y Humanidades por la Universidad Nacional de Quilmes, Argentina. 


\section{Resumen}

El presente trabajo destaca el valor de las etapas evolutivas tempranas en las cuales no solo se establecen las bases madurativas y neurológicas del desarrollo, sino que a partir de experiencias psicológicas, culturales y educativas precoces, se asientan los fundamentos para el desarrollo posterior de la persona. Desde esta premisa, se pone en discusión la importancia de generar políticas públicas intersectoriales para promover el desarrollo y la educación integral desde la primera infancia y se caracteriza la calidad de dichos servicios. Se comparten también algunas experiencias de países de América Latina.

\section{Palabras clave}

Educación Inicial, política educativa, educación integral, educación infantil.

\section{Introducción}

En este artículo me propongo abordar algunas cuestiones sobre la educación en el Nivel Inicial. En el marco de un reconocimiento a la importancia de este primer nivel educativo se discute sobre cómo entender esta etapa, integrando preocupaciones por cuidados tempranos, alimentación, salud, infraestructura, a preocupaciones sobre formación integral en saberes oportunos, socialización, juegos y lenguajes diversos. Esta perspectiva integral abarca la atención temprana de necesidades físicas, sociales, emocionales en el momento oportuno y con la participación de la familia. Se propone la generación de políticas públicas intersectoriales como una estrategia clave para desarrollar servicios para niños y niñas en América Latina. Finalmente, se ofrecen algunos ejemplos de cómo se está trabajando en la región.

\begin{abstract}
This article highlights the value of early developmental stages in which not only development and neurological bases are established, but from early psychological, cultural and educational experiences, the foundation is set for later development in a person. From this premise, I raise the question about the importance of generating intersectoral public policies to promote a comprehensive development and educational program from early childhood, where the quality of those services is emphasized. I also share some experiences in various Latin American countries.
\end{abstract}

\section{Keywords}

Early education, educational policy, integral education, preschool education.

\section{La importancia decisiva de la Educación Inicial}

Que los niños son el porvenir o que de la educación de las presentes generaciones dependen los futuros ciudadanos, no son frases trilladas y por eso, la educación de la primera infancia ha sido incluida en la mayoría de los procesos de transformación educativa en los países de todo el mundo. La implementación de cambios con el propósito de mejorar la calidad educativa de los mismos, es un desafío aún mayor porque:

Entendemos que el acceso, la permanencia y el egreso de la escuela infantil son condiciones necesarias, pero no suficientes, si no se oferta una propuesta de "buena enseñanza", que garantice la calidad de la experiencia educativa. Consideramos que, en este momento histórico, se nos impone la necesidad no solo 
de reafirmar la legitimidad de la Educación Inicial y su identidad pedagógica, sino también profundizar los modos de lograr un nuevo sentido para una verdadera democratización. (OMEP, 2012: 1)1

Las ramas de psicología, pedagogía, derecho y antropología (más recientemente) han investigado, teorizado y comprobado la importancia de las etapas evolutivas tempranas. En ellas, no solo se establecen las bases madurativas y neurológicas del desarrollo, sino que las experiencias psicológicas, culturales y educativas precoces serán el fundamento para todo desarrollo posterior. Con otros intereses de por medio, economistas, empresarios y políticos ven en los niños pequeños una inversión para logros de crecimiento económico y de capital humano.

Cada infante es un ser único, con características propias que lo definen como sujeto. Sin embargo, hay secuencias universales y predecibles en su desarrollo durante los primeros años de vida. Al tiempo que los niños se desarrollan y crecen, es esencial responder a sus necesidades básicas de salud, nutrición, protección y afectividad al tiempo que demandan diferentes tipos de propuestas educativas, interacción con objetos y otros seres humanos, constituir su subjetividad, ejercitar sus habilidades e ir desarrollando nuevas. Promover el desarrollo integral y garantizar un sano crecimiento desde las edades más tempranas supone entonces, buscar estrategias educativas, políticas de salud y protección, formas organizativas de Centros de Atención y Educación para que los estímulos brindados a los infantes propicien al máximo todas sus potencialidades.

Debemos entender que si hablamos de desarrollo hablamos de integralidad. En las fami-

1 El escrito fue firmado por la mayoría de los 1.300 docentes participantes del 5to Encuentro de Educación Infantil, realizado del 11 al 13 de mayo, 2012, llevado a cabo por la Organización Mundial para la Educación Preescolar (OMEP) en Buenos Aires, Argentina. La edición número cinco presentó como eje temático: "La Identidad Pedagógica de la Educación Infantil -0 a 6 años-: pilares y proyecciones". lias, la comunidad, las instituciones, las políticas públicas, hay una potencial conjunción para garantizar un escenario básico que permita una estimulación variada y equitativa para todos los niños y niñas. Metas Educativas 2021, documento de la XVIII Conferencia Iberoamericana de Educación celebrada en El Salvador el día 19 de mayo de 2008, asume entre sus objetivos prioritarios la atención integral a la primera infancia:

La meta que se plantea para el fin de la próxima década es que se multiplique el porcentaje de niños de 0 a 3 años que tienen posibilidades de acceso a experiencias educativas organizadas con esta finalidad y que se fortalezca el carácter educativo de todas las opciones existentes. (Marchesi, 2009: 8)

Afirmar que se requieren experiencias educativas organizadas es pensar en mucho más que condiciones básicas de alimentación, salud, cuidado y seguridad. Hay que transformar las modalidades centradas en la custodia y asistencia, a instituciones educativas que promuevan acciones integrales. No alcanza con planificar inversión económica, mejorar la infraestructura o generar espacios infantiles adecuados; hay que considerar experiencias y no solo cuidados, estimulación oportuna, de calidad y permanente, y no simplemente estímulos. Hay que considerar también que la constitución de la psiquis y el desarrollo de las inteligencias requieren de acciones eficaces, simbolizantes y continentes de cuidados cotidianos que otros seres humanos deben realizar con los bebés y los niños en una situación asimétrica, de poder, de saber y de organización del placer y del conocimiento, en contacto con esos sujetos. "Esta calidad en las intervenciones de los adultos no son indispensables desde el punto de vista biológico pero son la condición necesaria para que se constituyan los procesos de pensamiento" (Label, 2001). Reforzando la idea anterior y como dice Sandra Carli: "Es en la ligazón entre la experiencia de los niños y la institución de los adultos, 
que adviene el niño como sujeto" (citado por Mendioroz et al., 2009: 8).

La socialización, el juego, el lenguaje y el ambiente son clásicos y reconocidos pilares de la Educación Inicial. La socialización (apropiación de sentidos sociales de ser, de hacer, de constituirse como individuo social pleno), el juego (como lenguaje que se aprende y se enseña de forma privilegiada en el Nivel Inicial) (Sarlé 2008, 2012; Orezzio, 2012), el ambiente (como territorio vital que le permite al niño conocerlo, explorarlo y adentrarse en él con mayor autonomía) (Tallis, 2012), los lenguajes (verbales, no verbales, gestuales, artísticos) se refieren a los contenidos y experiencias que deben asumir docentes y cuidadores promoviendo desarrollo y aprendizajes en el transcurso de las experiencias que transitan los niños y niñas en los primeros años de sus vidas en instituciones educativas fuera del hogar.

Hoy se mantienen estos pilares, ejes que expresan lo que nos proponemos enseñar o los aprendizajes que esperamos sucedan en los niños pero se amplían y se recategorizan de otro modo en la propuesta de Frabboni (1986) y de Zabalza (2000) quienes coinciden en reconocer: la socialización o desarrollo personalsocial y la alfabetización cultural. Si ambos ejes se abordan en forma equilibrada diseñando propuestas que asuman la responsabilidad de enseñar contenidos vinculados con ellos, entonces se constituyen en las dimensiones centrales para lograr ofrecer una propuesta de Educación Integral para los niños. (Soto \& Violante, 2010: 4)

Estas autoras nos explican que el ambiente social, cultural, estético y físico se presenta como un universo a descifrar. Los lenguajes del ambiente también incluyen los lenguajes artístico-expresivos. El universo cultural se ofrece a los niños para iniciarlos en la alfabetización entendida en sentido amplio (no solo como el aprendizaje de la lectura y la escritura). El lenguaje verbal, hablar, escuchar, leer y escribir son desafíos centrales en estas edades. Al mismo tiempo que los niños se inician en el conocimiento y la comprensión del mundo (alfabetización cultural), es necesario fortalecer las posibilidades de establecer los vínculos con los otros, con los adultos y pares para aprender a confiar en las propias posibilidades de conocer, de explorar, de cantar, de pintar, de bailar, de jugar, de hablar con otros, de resolver problemas cotidianos, de saberse querido y respetado, de sentirse capaz de ser cada vez más autónomo. Es decir, es necesario enseñar contenidos vinculados con el desarrollo personal y social. Tal como afirma Carlos Cullen cuando escribe: "La educación, en su sentido más amplio, es una práctica social o un conjunto de prácticas sociales, consistente en socializar mediante la enseñanza de conocimientos" (2004:15).

En definitiva, para hacer efectiva la equidad y calidad educativa es importante trabajar en forma complementaria: el desarrollo personal y social y la alfabetización cultural. Cuando estos niños crezcan no solo querremos adolescentes, jóvenes y adultos sanos y bien alimentados, querremos sujetos-adultos-ciudadanos sanos psicológica y éticamente hablando, pensantes, críticos y creativos educativamente hablando, responsables y solidarios en el plano social y político.

\section{Servicios de desarrollo infantil}

Con los planteos anteriores, y sabiendo que en la infancia temprana se fijan bases para funciones superiores como el razonamiento y la memoria, el lenguaje, percepción, socialización y ciudadanía; todo servicio que atienda a los niños debe construirse y ofrecerse desde un enfoque de derechos, equidad, inclusión e interculturalidad en donde la prioridad -no solo en discurso de política pública sino en opción real- sean los pequeños. Si la prioridad son los niños, seres humanos en desarrollo pero integrales desde el inicio, los servicios deberán ser integrales.

En Jardines Maternales y Centros de Desarrollo infantil se debe ampliar la mirada para trabajar desde un enfoque de protección integral 
y de buena enseñanza, repensando las actividades y acciones como verdaderas instancias de aprendizaje significativos para los niños de estas edades, y como verdaderos aportes de docentes y cuidadoras desde la intencionalidad pedagógica. El docente se adapta a las posibilidades, tiempo, necesidades del niño y éste a su vez, se adapta a esos cuidados e intervenciones del adulto. Esta mutua adaptación es un proceso activo de parte de ambos y es lo que permite que se configure esta situación de enseñanza y aprendizaje. Es por esto que se requieren servicios de cuidado, atención y aprendizaje que:

- Ofrezcan potenciales y variados motivadores al desarrollo de las capacidades infantiles con el objetivo de formar personas libres de ser y para hacer como miembros de una sociedad: capacidades emocionales, cognitivas, lingüísticas y comunicativas, físicas para enfrentar, adaptarse y modificar entornos. Esto es, servicios que brinden experiencias ricas en juegos, estimulación, animación lectora, artes, expresión corporal favoreciendo el desarrollo de habilidades y capacidades motoras, sociales y creativas para promover el desarrollo integral.

- Ofrezcan y construyan siempre un entorno de buen trato, es decir, el bienestar, la salud y el desarrollo integral de los niños, a fin de que los niños crezcan sanos, que se promueva en ellos la solidaridad, la confianza, el cuidado, la amistad y el respeto a sí mismos y a los otros; para que a futuro sean adultos que, a su vez, respeten a los más pequeños.

- Articulen potencialidades de actores diversos: familia, Estado, comunidad, maestros y de ofertas coordinadas y articuladas de salud, educación, recreación y juegos, nutrición, atención a la diversidad a nivel intersectorial (empresas, instituciones estatales, privadas, etc.).

- Sean asequibles a todos los niños y niñas, incluyendo la educación intercultural bilingüe y a niños con necesidades educativas especiales.

- Ofrezcan acompañamiento y formación a padres y responsables a cargo de los destina- tarios (apoyos de salud, psicológicos, de nutrición, de formación, de visita a los hogares).

- Conformen lazos de sostén, confianza, respeto, complementariedad con el niño/niña y las familias.

- Atiendan las desigualdades educativas de origen social y familiar para favorecer una integración plena de todos los niños.

- Promuevan el aprendizaje y desarrollo de los niños como sujetos de derecho y partícipes activos de un proceso de formación integral, miembros de una familia y de una comunidad.

- Respeten y valoren las culturas de origen, sus lenguajes e historias personales y pautas para la crianza de los niños.

- Favorezcan el trabajo y la capacitación en equipo al interior de las instituciones.

- Estén dotados de espacios interiores y exteriores, dignos, seguros, adecuados, saludables, funcionales y equipados que favorezcan el juego, el aprendizaje, ambientes alfabetizadores y contextos ricos y motivantes.

\section{Políticas públicas y experiencias en la región}

Aguilar señala que una política pública es el diseño de una acción colectiva intencional, el curso que efectivamente toma la acción como resultado de las muchas decisiones e interacciones que comporta y, en consecuencia, los hechos reales que la acción colectiva produce (1996). Podemos agregar que las políticas públicas reflejan los valores que una sociedad afirma y dan prioridad a la hora de tomar decisiones o hacer inversiones (Kraft \& Furlong, 2006).

Entendemos aquí que la preocupación por la infancia compete al Estado, a la sociedad civil, a las familias, a instituciones formales y no formales, y por ende, la política pública involucra la participación de actores sociales diversos de la sociedad. Se espera que las políticas públicas como campo de abordaje interdiciplinario (ej. 
salud, desarrollo social, economía, educación, cultura y recreación) dialoguen para analizar, diseñar, planear, evaluar e implementar las acciones que reconozcan a los niños como destinatarios de servicios integrales y de calidad.

A la labor interdisciplinaria hay que sumarle la intersectorial entre gobiernos nacionales y provinciales, organizaciones de la comunidad, etc.; una gestión asociada para buscar alianzas y estrategias públicas y privadas y para promover organizaciones de base (ejemplo, entre mujeres, madres cuidadoras) con el objetivo de aumentar la capacidad de gestión y negociación. De esta forma se espera mejorar en calidad y sostenimiento, en el tiempo, para que haya más niños y niñas creciendo, desarrollándose y educándose sanos, seguros, en un medio contenedor y promotor de aprendizajes significativos para el presente y para el ejercicio de ciudadanía democrática y responsable.

Ya en 1968, el segundo Director Ejecutivo de UNICEF, Henry Labouisse, señaló una aspiración:

No deberían compartimentarse las necesidades del niño de acuerdo a las preocupaciones de un ministerio u otro, de un organismo $\mathrm{u}$ otro, o de tal o cual proyecto. El objetivo [de un enfoque intersectorial] consiste en aglutinar los conocimientos y las aptitudes de profesiones y disciplinas diferentes, y proporcionar servicios cuyos efectos a largo plazo se refuercen mutuamente. (citado por UNICEF, 2005:14)

Este siglo XXI exige un enfoque multidisciplinario e intersectorial para que inversiones e intervenciones -adoptando un enfoque de derechos- brinden un soporte apropiado a la familia y a las comunidades, prestando servicios de calidad que actúen sinérgicamente desde las políticas públicas.

Cuando UNICEF habla de las buenas prácticas en Hacia una política pública en desarrollo infantil temprano (2005) menciona algunas implicancias de un desarrollo infantil temprano integral:
- La supervivencia, el crecimiento y el desarrollo de los niños son mutuamente interdependientes.

- Las familias son responsables de la crianza de sus hijos y las naturales proveedoras de cuidado, afecto, estímulo y valores a los niños pequeños, de acuerdo a su cultura.

- Las oportunidades para que los niños logren mayores niveles de desarrollo personal aumentan cuando son favorecidas por políticas públicas y programas de apoyo a la familia formulados con abordajes multidisciplinarios e intersectoriales.

- La protección integral de la primera infancia, en el espíritu de la Convención sobre los Derechos del Niño, requiere implementarse mediante políticas públicas que garanticen su derecho a desarrollarse "hasta el máximo de su potencial".

- Las intervenciones a nivel local deben ajustarse a los criterios y estándares de calidad establecidos por los órganos rectores del sistema de protección integral, y fundarse en buenas prácticas.

- El desarrollo infantil temprano integral es un enfoque que orienta la adopción de medidas de políticas públicas que coordinen acciones a diferentes niveles, con el objetivo de mejorar:

- la calidad del cuidado familiar;

- el acceso de las familias a recursos, conocimientos, participación y servicios básicos de calidad;

- el apoyo de la comunidad y las instituciones a las familias.

A continuación se comparten de forma muy breve algunas experiencias en países de la región que muestran trabajos en pro de un desarrollo integral infantil y con estrategias de política pública intersectorial e interdisciplinaria: ${ }^{2}$

2 Los casos son tomados de UNICEF (2005), Hacia una política pública en desarrollo infantil temprano. Las buenas prácticas, Argentina. 
Argentina. Cemai (Centro Materno Infantil). Ciudad de Buenos Aires. El Cemai es un Centro de Acción Familiar dependiente del Departamento CAF, Dirección General de Niñez y Adolescencia, Gobierno de la Ciudad de Buenos Aires. Éste atiende a niños de la población perteneciente a la Villa 21/24 del barrio de Barracas. El proyecto constituye un modelo alternativo de educación no formal, que asigna a los miembros de la comunidad un papel protagónico. Crea e institucionaliza la figura de la "cuidadora de niños". La experiencia se basa en la integración de las cuidadoras a los equipos de profesionales, cuya función es facilitar y multiplicar nuevos aprendizajes.

Argentina. CIC (Centros Integradores Comunitarios). Río Gallegos, Provincia de Santa Cruz. Se trata de una política del Estado provincial orientada a descentralizar la atención a la salud, la educación inicial y la asistencia y promoción de familias vulnerables en un mismo espacio físico, debidamente equipado y con personal profesional y administrativo perteneciente a la planta provincial. Los jardines maternales y de infantes son atendidos por maestras y puericultoras. Se realizan visitas a los hogares, a través de las trabajadoras sociales. Se incluyen asimismo campañas educativas, grupos de autoayuda, proyectos de generación de empleo y la constitución de un Sistema Único de Identificación Social, con nodos en cada Centro.

Cuba. Programa "Educa a tu hijo". Este programa está centrado en la atención infantil en edades tempranas y forma parte del Sistema Cubano de Educación Inicial coordinado y reglamentado por el Ministerio de Educación a través del Subsistema de Educación Preescolar, que tiene dos modalidades: 1) la vía institucional (Círculos Infantiles y aulas de preescolar); 2) la vía no institucional, que incluye el programa "Educa a tu hijo" bajo la concepción de que en el proceso educativo es relevante el papel que se le asigna al adulto, principalmente en el ámbito familiar porque es quien realmente, por su posición y experiencia, ha de organizar, orientar y dirigir el proceso educativo de los niños, y definir qué deben lograr y cómo pueden alcanzarlo. La estructura organizativa del programa cuenta con diferentes niveles: Grupo Coordinador Nacional (elabora las políticas y está presidido por el Ministerio de Educación e integrado por representantes de distintos organismos públicos y no gubernamentales); grupos coordinadores a nivel provincial (definen las estrategias de trabajo atendiendo a cada realidad); grupos coordinadores municipales (ajustan las estrategias con criterio territorial, seleccionan a los promotores, trazan la política de capacitación y supervisan y evalúan la marcha del programa); grupos coordinadores a nivel de Consejo Popular (se encargan de materializar el proyecto con la participación activa y voluntaria de la comunidad). El programa recibe el apoyo de UNICEF.

En Cuba, la atención educativa por vía no institucional tiene carácter flexible y adopta distintas modalidades correspondiendo a la diversidad de contextos, como son:

- Atención individualizada: Orientación sistemática por el médico y la enfermera de la familia, durante el embarazo, para preparar a las madres y padres. Después del nacimiento y hasta los 2 años, se realizan visitas al hogar una o dos veces por semana, donde se orienta a la familia según los contenidos específicos volcados en los folletos del programa "Educa a tu hijo".

- Atención grupal: Para las edades de entre 2 y 6 años, se adopta la variante de "actividades conjuntas" en las que participan juntos las familias, sus niños y niñas y el personal orientador-ejecutor. Éstas pueden adoptar la forma de grupos diferenciados por tramos de edades y/o de grupos múltiples, en función de la complejidad de las tareas y su adecuación a las distintas necesidades infantiles.

Nicaragua y Honduras. Proyecto "Toma mi mano". Este programa cuenta con el apoyo de la Organización Internacional de Educación (OIE). Ha incorporado módulos de desarrollo infantil incluidos en el propio Programa de Alfabetización que ha sido elaborado por la 
Fundación Ser Humano de Buenos Aires. Dentro del proyecto, una de las líneas de trabajo que se contempla es la referida a la elaboración de un plan formativo sobre pautas de crianza a madres de bajos recursos que asisten a los círculos de alfabetización de adultos de Honduras y Nicaragua.

Chile. JUNJI (Junta Nacional de Jardines Infantiles). La Junta Nacional de Jardines Infantiles es una institución del Estado de Chile creada en 1970 como un estamento autónomo vinculado al Ministerio de Educación y cuyo fin es atender la educación inicial del país. Entrega educación parvularia de calidad a niños y niñas, preferentemente menores de cuatro años y en situación de vulnerabilidad social, para así generar las mejores condiciones educativas y contribuir a la igualdad de oportunidades. De este modo, la institución ayuda al desarrollo de las capacidades, habilidades y aptitudes de los párvulos y apoya a las familias a través de los programas de atención educativa en salas cuna y jardines infantiles administrados en forma directa y por terceros. Dentro del desarrollo de este compromiso, la JUNJI apunta a ofrecer una atención que considera la diversidad, conforme a una educación inclusiva, que contribuya a la equidad y a la enseñanza de valores como el respeto y la tolerancia. El trabajo inclusivo de la JUNJI beneficia con igualdad de oportunidades tanto a los párvulos como a la comunidad educativa en general, en cuanto los considera a todos sin distinciones para la construcción de una mejor sociedad.

Su estructura está organizada por un nivel central y por Direcciones Regionales que tienen como labor principal desarrollar las funciones que por ley le corresponde a la institución en cada región, representando a la Vicepresidenta Ejecutiva y haciendo cumplir los planes, programas, políticas y metas de la institución, a través de la coordinación de su quehacer con las autoridades regionales, provinciales y comunales. Históricamente, la JUNJI ha realizado la función de ser responsable de la calidad en la educación inicial a través de tres modalidades: la supervisión de los establecimientos de administración directa, tarea que tiene relación con dirigir los procesos educativos; la fiscalización de establecimientos de la sociedad civil a los que se transfieren fondos estatales; y el empadronamiento de salas cuna y jardines infantiles particulares. De manera permanente, la JUNJI evalúa en sus establecimientos cinco áreas de gestión (liderazgo, gestión de los procesos educativos, protección y cuidado, participación y compromiso de la familia, gestión y administración de recursos humanos), en las que radica el potencial promotor del aprendizaje organizacional y del mejoramiento continuo de la calidad del servicio entregado.

Estas pocas referencias ilustran algunas estrategias que la política pública puede utilizar para responder a las necesidades de los infantes sujetos de derecho: prestando servicios, impulsando acciones de forma centralizada o descentralizada, coordinando y evaluando, buscando alternativas de capacitación para quienes estén a cargo de los niños y niñas, entre otras posibles.

\section{Para terminar y seguir trabajando...}

El niño/a como sujeto de derecho es un ciudadano/a que merece y tiene el derecho a una educación que asegure su desarrollo personal y social desde el nacimiento. Esta perspectiva supone considerar a los niños como "beneficiarios" de derechos y programas asistenciales.

La educación infantil es un bien público y social, y por eso, las políticas sociales y educativas deben esforzarse en garantizar y ampliar el acceso al derecho de la educación. Pero no solo dar saltos cuantitativos, sino cualitativos desde la perspectiva integral aquí discutida.

Habrá que afanarse por articular ámbitos oficiales, interministeriales y multi-actorales con comunidades, organizaciones de la sociedad civil y del sector privado para ampliar una oferta de calidad apostando a un enfoque de protección integral en diversos formatos: escuelas infantiles, centros de desarrollo, jardines maternales, escuelas itinerantes, 
ludotecas, espacios de juego en hospitales, sala de cuna en empresas y otros proyectos y programas flexibles. Habrá que tejer y extender redes que habiliten espacios participativos y complementarios, de responsabilidad, obligaciones y derechos de cada actor: familias, comunidades, escuelas. Habrá que garantizar la formación y actualización continua de los educadores de bebés y niños para que puedan asumir la tarea docente como acompañante afectivo y transmisor cultural. Los pequeños esperan que los grandes no solo hablen de ellos y sobre ellos, sino que hagan grandes obras y construyan verdaderas políticas que les permitan crecer, aprender y tener voz propia, en un futuro cercano.

\section{Bibliografía}

Aguilar Villanueva, L.

1994. Estudio introductorio. En L. Aguilar Villanueva (Ed.), Problemas públicos y agenda de gobierno. México: Editorial Miguel Ángel Porrúa.

Aguilar Villanueva, L. 1996. Estudio Introductorio. En L. Aguilar Villanueva, La Hechura de las Políticas Públicas, 2da edición (p. 15-84). México: Miguel Ángel Porrúa.

Cabanellas, I., et al. 2005. Territorios de la infancia. Diálogo entre arquitectura y pedagogía. Barcelona: Editorial Cullen, C. Graó.

2004. Autonomía moral, participación democrática y cuidado del otro. Buenos Aires, Argentina: Ediciones Novedades Educativas.

Junta Nacional de Jardines Infantiles, JUNJI (Chile). Recuperado de http://www.junji.gob.cl/

Kaplan, D. H., \& Korinfeld, H. D. (Eds.). 2001. Derechos del niño, prácticas sociales y educativas, 8, 41. Buenos Aires: Novedades Educativas.

Kraft, M., \& Furlong, S.

2006. Public Policy: Politics, Analysis, and Alternatives, 2da edición. Washington, DC:

Label, C.
2001. En los tiempos de la constitución psíquica. Experiencias en instituciones que atienden niños menores de 2 años en situación de riesgo social. En Desarrollo cognitivo. La riqueza de un potencial sin límites. La educación en los primeros años. Argentina: Ediciones Novedades Educativas.

Marchesi, Á.

2009. Preámbulo. En J. Palacios, \& E. Castañeda, La primera infancia (0-6 años) y su futuro. Metas educativas 2021 (p. 7-9). España: Santillana/OEI.

Mendioroz, V., Druetta, A., \& Flores, S. 2009. Diseño Curricular Educación Inicial. Ciclo Jardín Maternal. Argentina.

Ministerio Bienestar Social-Ministerio Educación y Cultura-Nuestros Niños.

2004. Volemos alto. Claves para cambiar el mundo idale cinco minutos! Ecuador: Monsalve Moreno.

OMEP, Organización Mundial para la Educación Preescolar.

2012. Manifiesto de la OMEP por el Derecho a la Educación Integral y al Juego. Buenos Aires, Argentina: OMEP. Recuperado en http://www. omep.org.ar/article/manifiesto-de-la-omep/

Orezzio, M. F.

2012. Proyectos en juego. Experiencias infantiles, espacios y lugares para jugar. Juego y prácticas comunitarias. Buenos Aires: Fundación Navarro Viola.

Palacios, J., \& Castañeda, E. 2009. La primera infancia (0-6 años) y su futuro. Metas educativas 2021. España: Santillana/OEI.

Peralta, M. V.

2005. El currículo en el jardín infantil. Un análisis crítico. Chile: Editorial Andrés Bello.

Sarlé, P. M.

2012. Juego y educación infantil. Experiencias infantiles, espacios y lugares para jugar. Buenos Aires: Fundación Navarro Viola.

Sarlé, P. M. (Coord.).

2008. Enseñar en clave de juego. Enlazando juegos y contenidos. Argentina: Novedades Educativas.

Soto, C., \& Violante R.

2010. Didáctica de la educación inicial: Aportes para el desarrollo curricular. Buenos 
Aire, Argentina: Ministerio de Educación de la Nación.

Soto, C., \& Violante, R.

2001. Enseñar contenidos en el Jardín Maternal, una forma de compartir la crianza. Buenos Aires: Ediciones Paidós.

Tallis, J.

2012. Juego y Salud. Proyectos en juego: experiencias infantiles, espacios y lugares para jugar, 3. Buenos Aires: Fundación Navarro Viola.

UNICEF.

2005. Hacia una política pública en desarrollo infantil temprano. Las buenas prácticas (p. 14-15). Argentina.

Violante, R.

2008. Prólogo. En P. Sarlé, Enseñar en clave de juego. Buenos Aires: Ediciones Novedades Educativas.

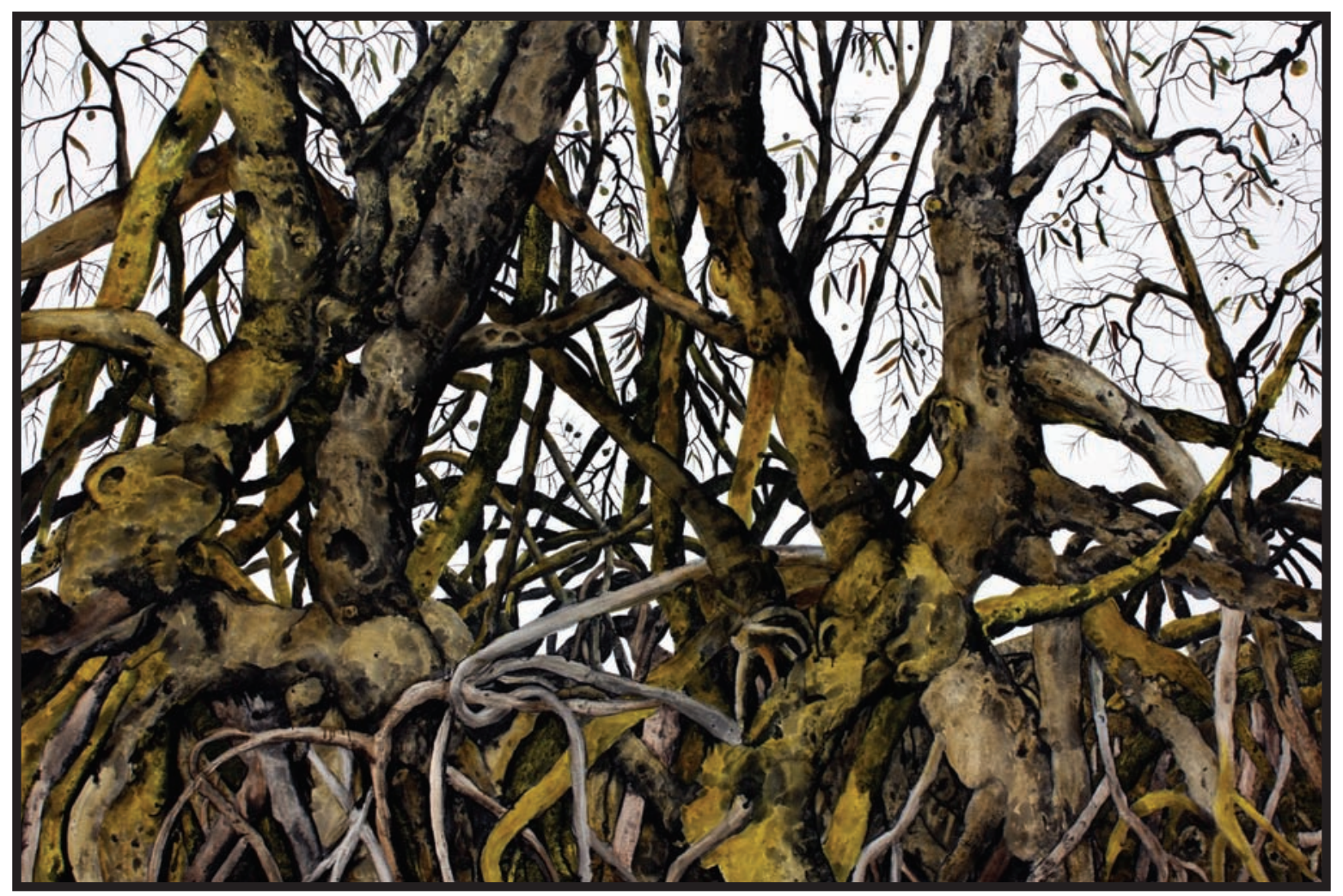

Manglar en Puerto Hondo. 150 x 210 cm 